\title{
BRAUER GROUPS OF RATIONAL FUNCTION FIELDS
}

\author{
BY BURTON FEIN ${ }^{1}$, MURRAY SCHACHER ${ }^{2}$, AND JACK SONN
}

Let $B(K)$ denote the Brauer group of a field $K$. The structure of $B(K)$ for $K$ a global field (that is, either an algebraic number field or an algebraic function field in one variable over a finite constant field) was completely determined in the 1930s by the work of Albert, Brauer, Hasse, and Noether [2, Chapter 7]. In view of this result it is natural to ask next for a description of $B(K)$ when $K$ is a function field over a global field. The purpose of this note is to announce a complete classification of the Brauer groups of rational function fields over global fields.

Before stating our main result we introduce the notation and terminology that we will use throughout this paper. $p$ will always denote a prime and if $G$ is an abelian torsion group, the $p$-primary component of $G$ will be denoted by $G_{p}$. We denote the $p$-primary component of the rationals mod 1 by $Z\left(p^{\infty}\right)$. For $G$ a group and $\alpha$ a cardinal number we denote the direct sum of $\alpha$ copies of $G$ by $\bigoplus_{\alpha} G$. We let $\omega$ denote the cardinality of the integers and we set $T_{i}=\bigoplus_{\omega}$ $C\left(2^{i}\right)$ where $C\left(2^{i}\right)$ is the cyclic group of order $2^{i}$. We denote the transcendence degree over $F$ of an extension $K$ of $F$ by t.d. $K / F$.

Let $F$ be a global field of characteristic $q \geqslant 0$. For $p \neq q$, we let $\epsilon\left(p^{t}\right)$ denote a primitive $p^{t}$ th root of unity in some algebraic closure of $F$. Define $\phi(F, p)$ to be the maximal $r$ such that for $p$ odd, $\epsilon\left(p^{r}\right) \in F(\epsilon(p))$, and for $p=$ 2, $\epsilon\left(2^{r}\right) \in F(\epsilon(4))$. Let $H(F, p, n)$ denote the abelian group with generators $x, y_{i}(i=\phi(F, p), \phi(F, p)+1, \ldots, k, \ldots)$ and relations $p^{n+1} x=0, p^{i} y_{i}=$ $x(i=\phi(F, p), \ldots)$. Let

$$
G(F, p)=\bigoplus_{\omega}\left[Z\left(p^{\infty}\right) \oplus \bigoplus_{n=0}^{\infty} H(F, p, n)\right] .
$$

By a rational function field over $F$ we mean a purely transcendental extension $K$ of $F$ with $1 \leqslant \mathrm{t} . \mathrm{d} . K / F<\infty$. With the above notation our main result is:

THEOREM. Let $K$ be a rational function field over the global field $F$ of characteristic $q \geqslant 0$. We have:

(1) if $q>0$, then $B(K)_{q} \cong \bigoplus_{\omega} Z\left(q^{\infty}\right)$;

Received by the editors May 15, 1979.

AMS (MOS) subject classifications (1970). Primary 16A40; Secondary 12A50.

Key words and phrases. Brauer group, class group, global field.

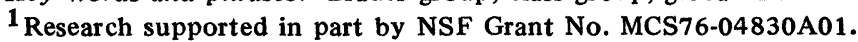

2 Research supported in part by NSF Grant No. MCS77-01725. 
(2) for $p \neq q$, if either $p$ is odd or $p=2$ and $\epsilon(4) \in F$, then $B(K)_{p} \cong$ $G(F, p)$;

(3) for $q=2$ and $\epsilon(4) \notin F, B(K)_{2} \cong G(F, 2) \oplus T_{1}$.

This result has a surprising consequence:

COROLlaRY. Let $K$ and $E$ be rational function fields over $F$. Then $B(K)$ $\cong B(E)$.

Maintaining the context of the theorem, suppose next that $[L: K]<\infty$. If $p \nmid[L: K]$, then an easy cohomological argument shows that $B(L)_{p} \cong B(K)_{p}$ $\oplus$ ker Cor $(B(L))_{p}$ where ker Cor $(B(L))_{p}$ denotes the kernel of the corestriction map from $B(L)_{p}$ to $B(K)_{p}$. Thus the theorem also gives information even in the algebraic function field case. (When $p \mid[L: K]$, information about $B(L)_{p}$ can also be deduced from [3, Theorem 3].)

We sketch a proof of the theorem. In [1] (see also [3, Proposition 1]) Auslander and Brumer prove that if $F$ is an arbitrary field of characteristic $q \geqslant$ 0 and $t$ is transcendental over $F$, then for $p \neq q$,

$$
B(F(t))_{p} \cong B(F)_{p} \oplus \bigoplus_{f} X(F[t] /(f(t)))_{p}
$$

where $f$ runs through all monic irreducible polynomials in $F[t]$ and where $X(E)$ denotes the continuous character group of $E$. Thus $B(F)_{p}$ is a direct summand of $B(K)_{p}$ for $p \neq q$. Using the structure of $B(F)_{p}$ as determined by Albert, Brauer, Hasse, and Noether, we determine the maximal divisible subgroup $D B(K)_{p}$ of $B(K)_{p}$ for $p \neq q$. For $p=q, B(K)_{q}$ is divisible and its structure is also easily determined. Since $D B(K)_{p}$ is a direct summand of $B(K)_{p}$ we are reduced to determining $R B(K)_{p}=B(K)_{p} / D B(K)_{p}$. Since $B(K)_{p}$ is easily shown to be countable, $R B(K)_{p}$ is classified by its Ulm invariants [6, page 27]. To determine the exact values of the Ulm invariants it is necessary, in view of the theorem of Auslander and Brumer, to compute Ulm invariants for the various character groups that arise. These Ulm invariants can be computed using class field theory when t.d. $K / F=1$. For finite ordinals, these invariants are computed in this special case by the first two authors in [3]; the determination of the remaining Ulm invariants in this case is reduced to the proof of an interesting conjecture about class groups in global fields. Settling this conjecture, the third author proves the following in [7]:

THEOREM. Let $F$ be a global field, $n$ a positive integer not divisible by the characteristic of $F$. Then there exists a finite (solvable) extension $E$ of $F$ having the following property: for each prime $p$ dividing $n$ there is a cyclic extension $L$ of $E$ of degree equal to the exact power of $p$ dividing $n$ which is unramified at all primes of $E$. If $M$ is any cyclic extension of $E$ of p-power degree larger than that of $L / E$ and such that $M \cap L \neq E$, then some finite prime of $E$ 
not dividing $p$ ramifies in $M$. In particular, the class group of $E$ has a cyclic direct summand of order $n$.

The fields $L$ and $E$ of this theorem can be constructed as follows: Let $F^{\prime}$ $=F\left(\epsilon\left(n^{2}\right)\right)$ and let $S$ be a finite set of primes of $F^{\prime}$ containing the divisors of $n$ and large enough so that the $S$-class number of $F^{\prime}$ is one. Let $F^{\prime \prime}$ be the extension of $F^{\prime}$ generated by all $n$th roots of $S$-units of $F^{\prime}$ and let $P$ be a prime of $F^{\prime}$ outside of $S$ which splits completely in $F^{\prime \prime}$. Let (, ) denote the $n$th power norm residue symbol in $F_{P}^{\prime}$. There exist $n$-hyperprimary, relatively prime elements $\pi, \rho$ in $F^{\prime}$ such that $(\pi, \rho)=\epsilon(n)$. Then $E=F^{\prime}\left((\pi \rho)^{1 / n}\right)$ and $L=$ $F^{\prime}\left(\pi^{1 / n}, \rho^{1 / n}\right)$. (We remark that Yahagi [8] has recently proved that if $n$ is a power of a prime $p$ and $F$ is a number field of class number prime to $p$, then, for any abelian group $G$ of order $n$, there exists an extension $E$ of $F$ such that the $p$-Sylow subgroup of the class group of $E$ is isomorphic to $G$.)

Using essentially geometric arguments, the first two authors in [5] show that the Ulm length of $R B(K)_{p}$ is independent of the particular rational function field $K$. When t.d. $K / F=1$, the result stated above implies that the Ulm invariants of $R B(K)_{p}$ at infinite ordinals are all $\omega$. In the general case, since $K$ contains a subfield $L$ with t.d. $L / F=1$, the theorem of Auslander and Brumer together with the results above implies that all $\mathrm{Ulm}$ invariants at infinite ordinals of $R B(K)_{p}$ equal $\omega$. The Ulm invariants at finite ordinals of $R B(K)_{p}$ are determined in [4] and [7]. Once the full set of Ulm invariants of $B(K)_{p}$ has been determined, the proof of the theorem is completed by showing that the groups appearing in the statement of the theorem have the required Ulm invariants and the appropriate maximal divisible subgroups.

\section{REFERENCES}

1. M. Auslander and A. Brumer, Brauer groups of discrete valuation rings, Nederl. Akad. Wetensch. Proc. Ser. A 71 (1968), 286-296.

2. M. Deuring, Algebren, Springer-Verlag, New York, 1966.

3. B. Fein and M. Schacher, Ulm invariants of the Brauer group of a field, Math $\mathrm{Z}$. 154 (1977), 41-50.

5. - Brauer groups and character groups of function fields, J. Algebra (to appear).
4. - Ulm invariants of the Brauer group of a field. II, Math. Ž. 163 (1978),

6. I. Kaplansky. Infinite abelian groups, University of Michigan Press, Ann Arbor, Mich., 1956.

7. J. Sonn, Class groups and Brauer groups, Israel J. Math. (to appear).

8. O. Yahagi, Construction of number fields with prescribed l-class groups, Tokyo J. Math. 1 (1978), 275-284.

DEPARTMENT OF MATHEMATICS, OREGON STATE UNIVERSITY, CORVALLIS, OREGON 97331

DEPARTMENT OF MATHEMATICS, UNIVERSITY OF CALIFORNIA, LOS ANGELES, CALIFORNIA 90024

DEPARTMENT OF MATHEMATICS, TECHNION-ISRAEL INSTITUTE OF TECHNOLOGY, TECHNION CITY, HAIFA, ISRAEL 